\title{
Determinants of happiness among healthcare professionals between 2009 and 2019: a systematic review
}

\author{
Rose Nabi Deborah Karimi Muthuri(iD ${ }^{1 凶}$, Flavia Senkubuge ${ }^{1} \&$ \\ Charles Hongoro $1,2,3,4$
}

Happiness is characterized by experiencing positive emotions while simultaneously perceiving one's life as meaningful and worthwhile. Research on the mental health of healthcare professionals usually focuses on psychopathology as opposed to the positive aspects of mental health. Thus, the purpose of this study is to examine recent literature on individuallevel and organization-level determinants of healthcare professionals happiness. The EBSCOhost, PubMed, Scopus, and Web of Science databases were searched for articles published between 2009 and 2019 that focused on happiness among healthcare professionals. Based on the eligibility criteria, both quantitative and qualitative studies were selected for this systematic review. The final review included a total of 18 studies. The main measures of healthcare professionals happiness in cross-sectional and intervention studies were selfreport measures, and the main measures of happiness in qualitative studies were interviews. Multiple determinants of healthcare professionals happiness were identified in this systematic review. The interplay between individual-level and organization-level determinants collectively contributes to the happiness of healthcare professionals. Existing evidence highlights the importance of healthcare professionals happiness in the strengthening of the healthcare workforce and healthcare systems. This review also highlights the importance of health policy makers, health authorities and healthcare professionals in creating and effectively implementing policies and strategies to increase healthcare professionals happiness. Future similar studies should use large and more heterogeneous samples of healthcare professionals from various settings to generate contextually relevant and robust evidence regarding methods to enhance healthcare professionals happiness and ultimately the performance of health systems globally.

\footnotetext{
${ }^{1}$ School of Health Systems and Public Health (SHSPH), Faculty of Health Sciences, University of Pretoria, Pretoria, South Africa. ${ }^{2}$ Developmental, Capable and Ethical State Division, Human Sciences Research Council (HSRC), Pretoria, South Africa. ${ }^{3}$ Faculty of Science, Tshwane University of Technology,

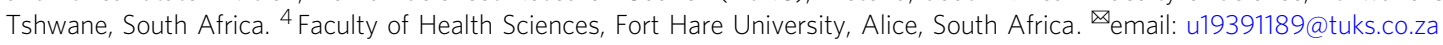




\section{Introduction}

or centuries, happiness has been viewed as an essential component in human life (Pollock et al., 2016). Ancient Greek philosopher Aristotle (384-322 BC) believed that happiness was the ultimate motivator of peoples' choices and actions (Pollock et al., 2016). Additionally, the United Nations (UN) has recognized the pursuit of happiness as an important human goal (Paiva et al., 2016). On July 12, 2012, the United Nations General Assembly comprising of all 193 presidents of the UN member states, passed a resolution (A/Res/66/281) declaring every 20 March the International day of happiness; this was following the authorization of a prior resolution (A/Res/65/281) titled, Happiness: towards a holistic approach to development (United Nations, 2012). Both resolutions encourage policy makers to aim to achieve and enhance happiness and well-being when creating or modifying policies (United Nations, 2012). Since 2012, the United Nations Sustainable Development Solutions Network (UNSDSN) has published the World Happiness Report (WHR) and has ranked countries according to the World Happiness Index annually.

The UN and various researchers have recognized the significance of happiness not only as an individual endeavor but also as a public endeavor (Paiva et al., 2016). Veenhoven explains the importance of governments applying a utilitarianism approach to happiness (Veenhoven, 2017). A utilitarianism approach to happiness means authorities should aim to achieve greater happiness for the greater numbers within their populations (Veenhoven, 2017). Basing happiness policy on factual and logical reasoning such as research findings will potentially result in individuals being more optimistic about living and working towards a better life (Veenhoven, 2017). Thus, it is essential that policy creation and the implementation of effective strategies pertaining to happiness be based on contextually relevant characteristics (Paiva et al., 2016).

Positive psychology theorists believe that research and policy needs to move from solely focusing on psychopathological aspects, i.e., individuals' weaknesses, to optimizing the positive aspects, i.e., individuals' strengths (Seligman and Csikszentmihalyi, 2000). There are several definitions of happiness as a construct, and these definitions generally imply the experience of a positive state of emotion (Paiva et al., 2016). Positive psychologists advocate for the understanding of how an individual's happiness and well-being influence his or her productivity and optimal functionality to fully achieve his or her potential in life (Seligman and Csikszentmihalyi, 2000). Happiness is an attitudinal phenomenon, meaning one's perceptions and beliefs of happiness influence the actual experience of happiness (Veenhoven, 2017; Joshanloo et al., 2017).

Happiness is the hallmark of positive affect, which entails both experiencing positive emotions in conjunction with perceiving one's life as virtuous, meaningful and worthwhile (Berkland et al., 2017; Walsh et al., 2018; Lyubomirsky et al., 2005). In 2017, the WHR reported that happiness at work is associated with increased productivity and consistency (Jan-Emmanuel De Neve, 2017). The World Health Organization (WHO) Health System Framework consists of six building blocks, one of which is the health workforce (World Health Organization, 2007). In 2012, researchers stated that the health workforce is at the core of every health system (Anand and Bärnighausen, 2012). Healthcare professionals are part of the health workforce. Healthcare professionals are individuals who are formally trained in providing healthcare services to human beings that are curative, preventive, and/or promotive in nature (World Health Organization, 2013).

Nevertheless, mental health research among healthcare professionals within health systems is largely focused on reporting their weaknesses and pathologies as opposed to positive aspects of their mental health. For instance, there is more evidence on healthcare professionals experiences of higher levels of distress, burnout syndrome, depressive symptoms, and suicidal ideations more than among other professionals in other sectors (Brand et al., 2017; Kokonya et al., 2014; Dyrbye et al., 2017; Lafreniere et al., 2016). It is essential to recognize problems and challenges, but it is equally important to promote the optimistic aspects of mental health. Although positive attitudes and experiences may not eliminate difficult experiences, they act as a buffer that is paramount for healthcare professionals (Einarsdottir, 2012).

In 2018, scholars reported that happiness is a significant factor of health system efficiency (See and Yen, 2018). Recently, empirical studies assessing and reporting happiness among healthcare professionals have been increasing. Hence, this study aims to systematically review recent literature to determine the role of individuals and organizations in healthcare professionals happiness. Systematic reviews are important tools used for summarizing evidence in a precise and reliable way (Liberati et al., 2009). Using a systematic review, the purpose of this study was to examine the individual and organizational determinants of healthcare professionals happiness. Specifically, we performed a narrative synthesis of recent literature between 2009 and 2019.

The main research questions of this study were, 'What are the individual and organizational determinants of healthcare professionals happiness and how can they be applied in happiness-related policies and strategies?' Though there have been various empirical studies on healthcare professionals happiness, to the best of our knowledge, there is no systematic review reporting or answering this research question. This systematic review will also provide up-to-date information for decision makers to make informed decisions regarding healthcare professionals happiness behavioral interventions (Liberati et al., 2009). Based on the belief that we can achieve "... better health outcomes through better informed decisions.” p. 1040 (Atkins et al., 2005), this systematic review aims to summarize recent literature in order to inform policy makers and to identify the significant roles of individuals (i.e., healthcare professionals) and organizations (e.g., health facilities and the national health systems) in enhancing healthcare professionals happiness. Happiness-related studies have improved and informed decision-making processes in terms of policies and regulations based on legal, organizational and national happiness interventions (Huang, 2010). Finally, this review identifies research gaps and provides for future directions for studies in this area.

\section{Methods}

The protocol of this systematic review was registered in advance, and the PROSPERO registration number is CRD42020145855. The protocol is available at elsewhere ${ }^{1}$.

This systematic review was conducted in accordance with the Preferred Reporting Items for Systematic Review and MetaAnalyses (PRISMA) guidelines (Moher, 2015). The results of the systematic selection process are illustrated in a PRISMA flowchart that is shown in Fig. 1 (Moher, 2015). Additionally, to ascertain the quality of this systematic review, we applied the guidelines provided by the Risk of Bias Assessment Tool for Systematic Reviews (ROBIS). The ROBIS has been used to assess and strengthen systematic reviews in healthcare-related fields that influence health policy in specialties such as public health and quality of health care, among others (Whiting et al., 2016).

Eligibility criteria. The selection process of this systematic review was based on eligibility criteria for both inclusion and exclusion. The inclusion criteria included selecting empirically 


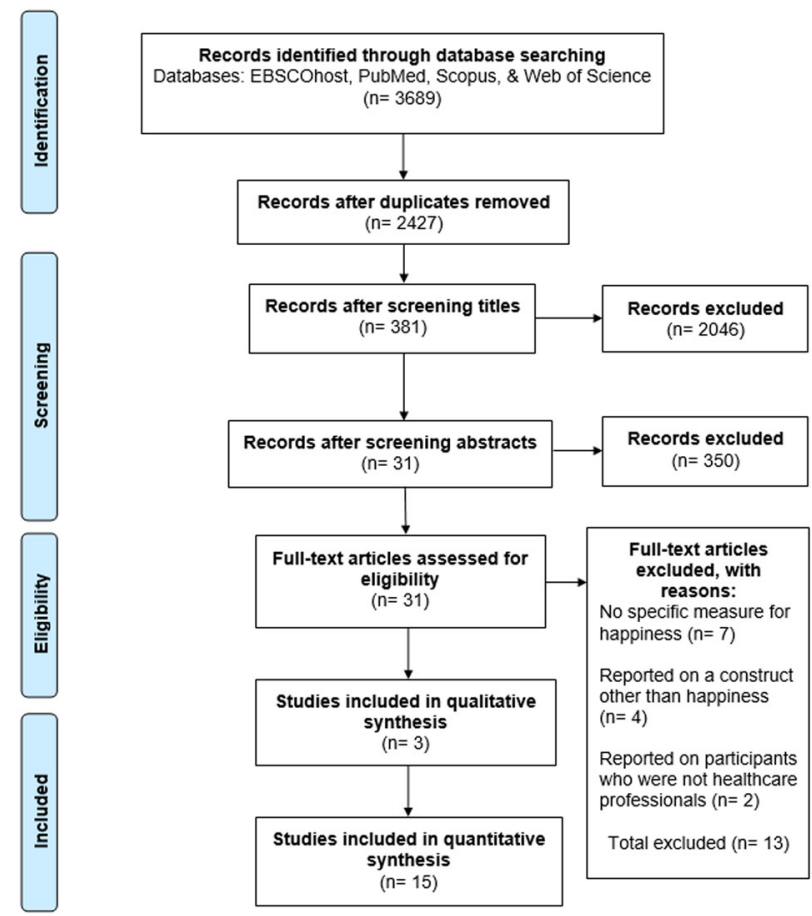

Fig. 1 The PRISMA flow diagram for the systematic review of the determinants of healthcare professionals happiness. As adapted from source: Moher (2015).

peer-reviewed studies that report on the determinants or factors of healthcare professionals happiness that were published in the English language between 2009 and 2019, and the studies must have clearly indicated the measure of happiness. Focusing on this 10-year period allowed us to examine recent literature on healthcare professionals happiness in this systematic review. This was essential because systematic reviews are among the ideal sources of evidence-based health policy, and they often use recent literature to facilitate relevant, informed decision making (Shojania et al., 2007). Since there is currently no gold standard tool for measuring happiness, various tools were used in the included studies (Paiva et al., 2016). Both quantitative and qualitative studies were reviewed.

Exclusion criteria included nonempirical studies, reviews, gray literature, non-peer-reviewed studies, studies that were not published in the English language, and studies not published between 2009 and 2019. In addition, studies on happiness among workers in other sectors, students and non-medically trained caregivers were also excluded. Additionally, studies with no specific measure for happiness were excluded. Studies that claimed to assess happiness then reported on a different construct, even ones related to mental health, were also excluded.

The PRISMA flow diagram shown in Fig. 1 presents the number of articles included and excluded during the selection process as per the PRISMA statement and study eligibility criteria (Moher, 2015).

Number of studies included by country. The eleven countries represented in this review included the United States of America (USA) ( $n=7$ studies), Iran ( $n=2$ studies), Iceland ( $n=1$ study), Brazil ( $n=1$ study), India ( $n=1$ study), Romania ( $n=1$ study), South Africa ( $n=1$ study), Canada ( $n=1$ study), Croatia ( $n=1$ study), Thailand ( $n=1$ study), and Spain ( $n=1$ study) (see Fig. 2).

\section{Number of articles included per country}

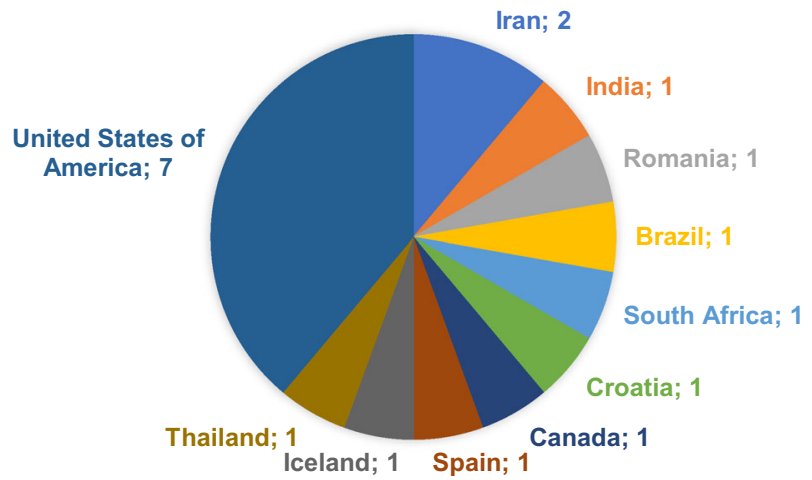

Fig. 2 Number of articles included per country. Source: Authors own.

Data sources and search strategy. The databases used to systematically search for relevant articles were EBSCOhost, PubMed, Scopus, and Web of Science. These databases are credible platforms that are sources of scholarly and peer-reviewed information with search tools that enable access to dates of coverage and provide a platform to identify studies in a systematic way (Liberati et al., 2009). These databases were selected because they contain literature related to various disciplines of health, including healthcare professionals, and health policy. Therefore, through these four databases, we obtained scholarly data from studies on our topic of focus from reliable researchers and experts.

The search terms used were "happiness" AND "healthcare professionals", "healthcare workers", "health personnel", "happy doctors", "happy physicians", and "happy nurses". A multidisciplinary search using various combinations of keywords and MeSH terms was used and differed accordingly between databases. Boolean operators such as "AND" and "OR" were applied to accommodate different terminology related to our review.

From the databases, citations were exported into Endnote $\mathrm{X}^{\mathrm{m}}$ (Clarivate Analytics, Philadelphia, PA, United States of America), a referencing manager software. Duplicates were removed on Endnote $\mathrm{X}^{\mathrm{m}}$. Articles that failed to meet the inclusion criteria were excluded. The final selection of the articles was based on the independently selected articles and approved by the review team.

Data extraction. Publication metadata were extracted using a data extraction template that was created and modified according to all the studies reviewed. The publication details were captured and summarized in a tabular format developed by the authors of this review. The publication metadata were as follows: author(s), year of publication, country of study, main goal of study, sample size, measure of happiness, statistical procedure used and main findings of the reviewed studies. Discrepancies were discussed and resolved through discussion by the review team.

Risk of bias. Using the Joanna Briggs Institute (JBI) Critical Appraisal Checklist tools, we performed quality assessments on the risk of bias for each study in this systematic review (Joanna Briggs Institute). The quality assessment was performed according to the study design of each study reviewed, such as crosssectional studies, cohort studies, randomized controlled trials and qualitative research studies. After assessing the risk of bias, the review team selected a total of 18 studies. 


\section{Results}

Overview. In this review, based on a systematic search of the Scopus, PubMed, EBSCOhost, and Web of Science databases, 3689 records were identified. The 3689 records were exported into Endnote $\mathrm{X} 8{ }^{\mathrm{TM}}$, and 1262 duplicate records were eliminated, leaving 2427 records. After screening the titles, 2046 records were excluded, leaving 381 records. After screening the abstracts, 31 records remained. After full-text screening, 13 records were excluded. The reasons for excluding studies were either lack of a specific measure for happiness $n=7$, reporting a different construct other than happiness $n=4$ or reporting on participants who are not healthcare professionals $n=2$. In total, 18 studies were eligible for this review, including 15 quantitative studies and 3 qualitative studies (see Fig. 1 and Tables 1-3). The total number of healthcare professionals represented across all articles included was $N=3501$.

Of the 18 eligible studies included in this review, six different measures of happiness were identified in the quantitative studies. The Subjective Happiness Scale (SHS) (Lyubomirsky and Lepper, 1999) was used in six studies to assess overall happiness on a 7point Likert-type scale. The Oxford Happiness Questionnaire (OHQ) (Hills and Argyle, 2002) was used in three studies to measure happiness on a 6-point Likert scale. The Oxford Happiness Inventory (OHI) (Argyle et al., 1989) assessed happiness in two studies on a 6-point Likert scale. The Happiness Measure Questionnaire (Appel et al., 2013) was used in one study to assess happiness with a 10-point scale. The Pemberton Happiness Index (PHI) (Hervás and Vázquez, 2013) measured happiness in one study with an 11-point Likert scale. Two studies used a single item to measure happiness; one study used an item with a 5-point Likert scale (Petriş et al., 2013), and the other used an item with a 10-point Likert scale (Prizmic et al., 2009). The three remaining studies that applied a qualitative approach used interviews and/or participant observations to measure happiness.

Methodological quality. As shown in Table 4, all 18 reviewed studies underwent methodological quality assessments and were of high quality due to their low risk of bias. Nonetheless, two studies with unclear critical appraisal outcomes were found, due to confounding variables. Nevertheless, these issues were appropriately addressed in the multivariate analysis performed in both quantitative studies.

Determinants of healthcare professionals happiness in the cross-sectional studies. In this systematic review, there were eleven cross-sectional studies that included approximately $86.1 \%$ of the total number of healthcare professionals $(n=3014 / 3501)$. The types of healthcare professionals represented in the crosssectional studies included counselors (Chaverri et al., 2018), cardiologists (Petriş et al., 2013), dentists (Kaipa et al., 2017), nurses (Abdollahi et al., 2014; Khosrojerdi et al., 2018), physiotherapists (Elliot et al., 2018), psychiatrists (Machado et al., 2016), physicians (family, general and fellowship graduate) (Eckleberry-Hunt et al., 2016; Duffrin and Larsen, 2014), and general healthcare workers (Prizmic et al., 2009; Benzo et al., 2017). The measures used in the cross-sectional studies to assess healthcare professionals happiness included the OHI, the OHQ, the SHS and single-item measures, as presented in Table 1.

In Iran, moderate happiness was reported both in 2014 among 252 nurses (Abdollahi et al., 2014) and 2018 among 422 nurses (Khosrojerdi et al., 2018). In 2014, nurses reported higher levels of happiness among nurses with greater hardiness (the ability to adapt oneself in problematic life situations) (Abdollahi et al., 2014). Lower scores of perceived stress result in higher happiness scores among nurses, which was depicted by the negative correlation between stress and happiness scores (Abdollahi et al., 2014). Four years later, in 2018, another study among nurses in Iran reported that $50.3 \%$ of the variance in happiness was attributed to 10 determinants (Khosrojerdi et al., 2018). The determinants included monthly income, satisfaction with income, job satisfaction, quality of life, current hospital ward, length of working in the current ward, work shift, age and satisfaction with physicians' conduct and performance (Khosrojerdi et al., 2018). Among the 10 determinants, the most significant determinant was satisfaction with mental health, which explained $13 \%$ of the variance in nurses' happiness (Khosrojerdi et al., 2018).

Average to moderately high scores of happiness were reported in India, Romania and South Africa across different types of healthcare professionals. In India, moderately high scores of happiness were reported among dentists $(n=194)$, with the most important determinant of happiness being the type of profession. The dentists who were both clinicians and academicians reported experiencing more happiness than the dentistry professionals who were either clinicians or academicians (Kaipa et al., 2017). In Romania, among cardiologists $(n=187)$, an average level of happiness was reported (Petriş et al., 2013). The determinants of happiness among the cardiologists included age, number of vacation days per year and single marital status (Petriş et al., 2013). Among physiotherapists $(n=395)$, moderately high scores of happiness were reported (Elliot et al., 2018). Approximately $60 \%$ of the variance in physiotherapist happiness was explained by high scores on four determinants, namely, life balance, leisure, influence and optimism (Elliot et al., 2018).

Among mental healthcare professionals, specifically counselors $(n=153)$ in the United States of America (USA) and physiatrists in Brazil $(n=104)$, moderate to moderately high scores of happiness were reported. In 2018, among counselors, higher happiness scores were predicted by an increased number of sessions per client and low severity of posttraumatic stress symptoms experienced by the counselor (Chaverri et al., 2018). In Brazil, researchers reported that $50.2 \%$ of their happiness was explained by the following determinants: having children, good quality sleep and the use of three defense styles related to mature factors, namely, anticipation, humor, and the use of neurotic factors (e.g., annulment) (Machado et al., 2016). Hence, the less psychiatrists reported using the defense mechanism of acting out (an immature factor) in their profession, the happier they were (Machado et al., 2016).

Two studies in the USA reported moderately high happiness scores among family physicians. In 2014, general family physicians reported lower scores of happiness compared to fellowship-trained family physicians (Duffrin and Larsen, 2014). The type of physician training as a determinant of happiness explained $10 \%$ of the variance in happiness scores (Duffrin and Larsen, 2014). In addition, gender differences were found, such that male family medicine physicians reported significantly higher happiness scores than their female counterparts (Duffrin and Larsen, 2014). In 2016, among family physicians $(n=449)$, happiness was significantly determined by four factors, including experiencing a sense of career purpose, a sense of personal accomplishment, less distress and a higher perceived ability to manage their workload experience (Eckleberry-Hunt et al., 2016). Workload manageability among family physicians explained $\sim 35.8 \%$ of their subjective happiness (Eckleberry-Hunt et al., 2016).

In the USA, scholars examined two determinants of healthcare workers' $(n=400)$ happiness, namely, self-compassion and weekly exercise (Benzo et al., 2017). These factors explained $\sim 39 \%$ and $40 \%$ of the variance in healthcare workers' happiness, respectively (Benzo et al., 2017). In Croatia, average scores of overall happiness were reported, and age was the only noteworthy 


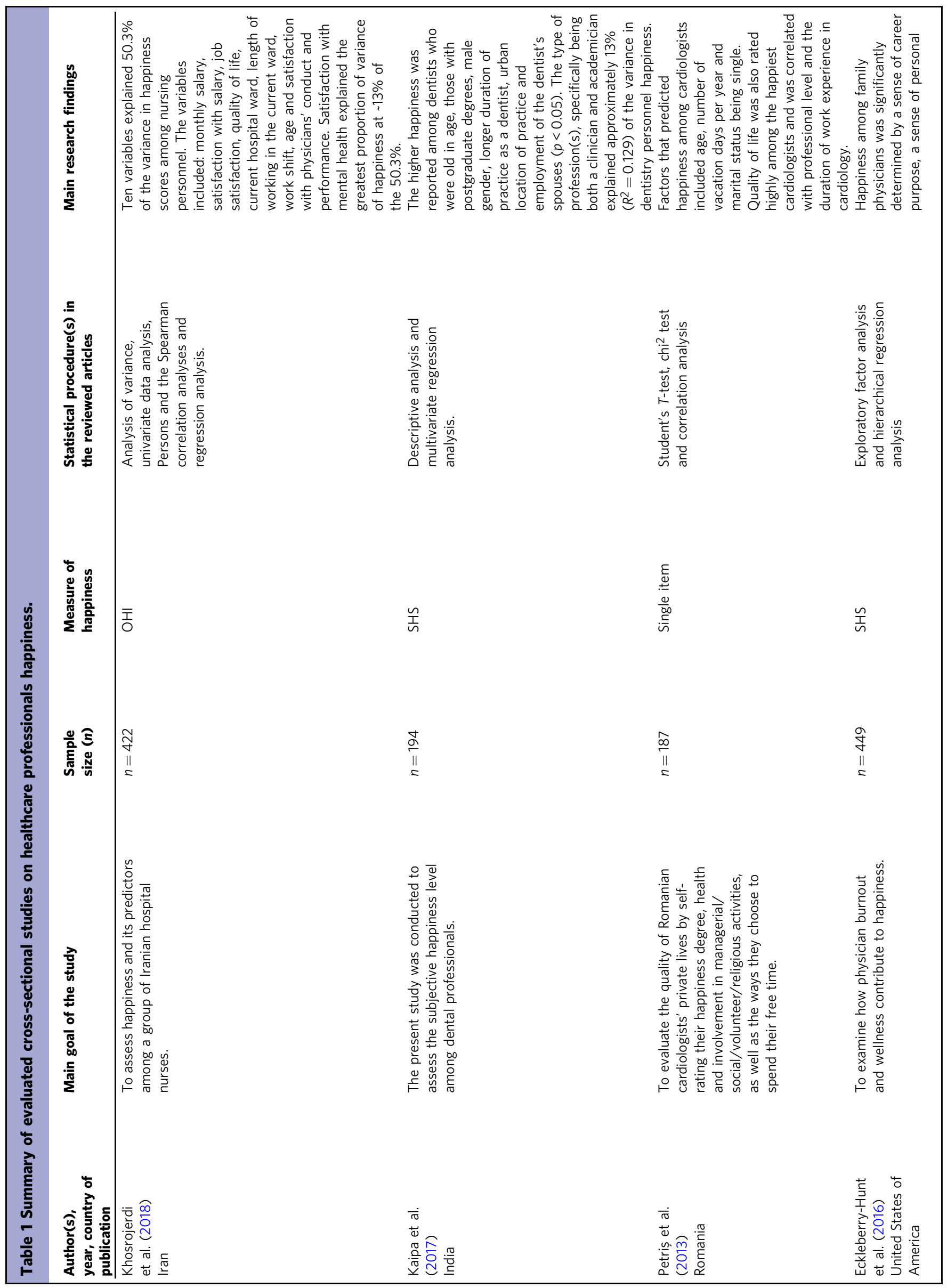




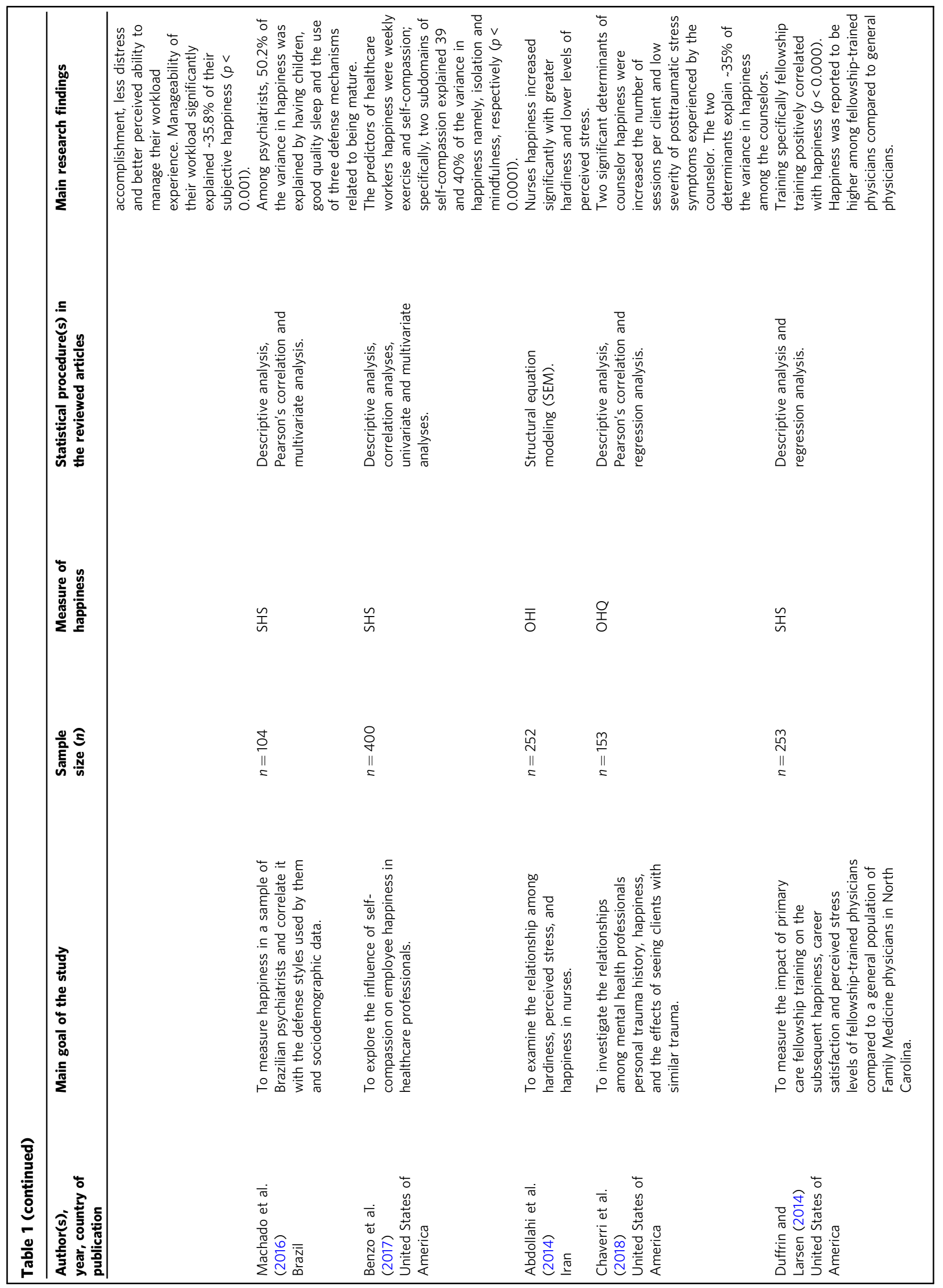




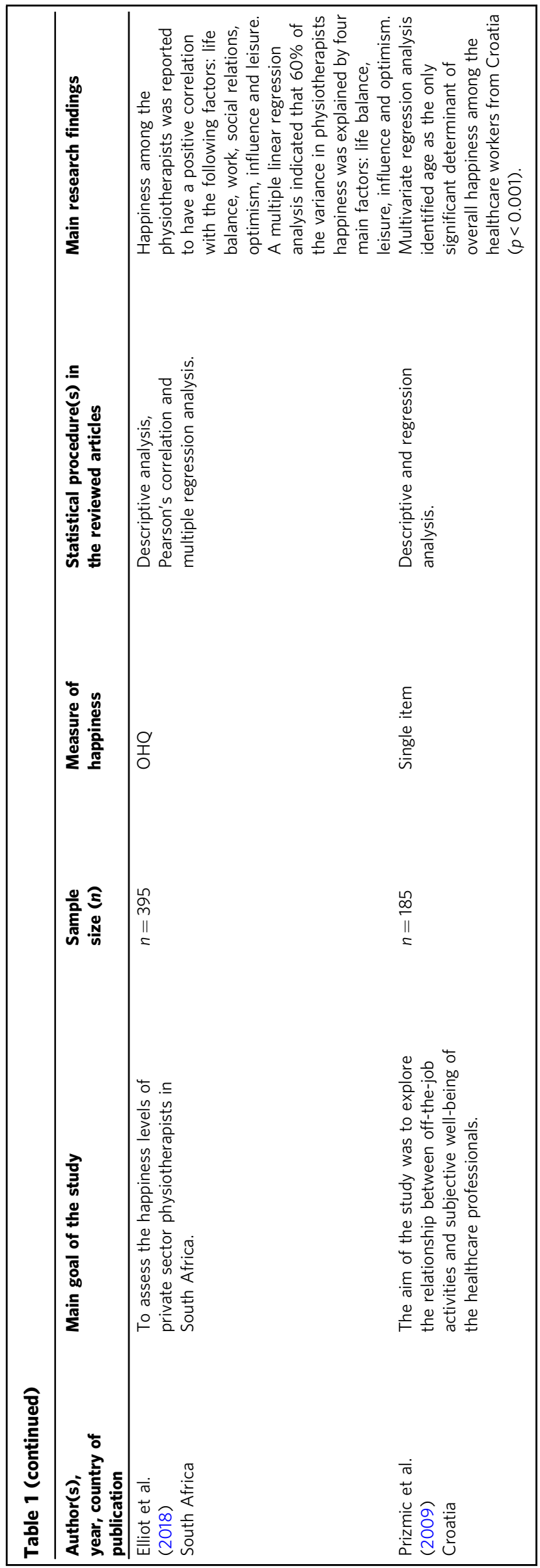

determinant of overall happiness among the healthcare workers $(n=185)$ (Prizmic et al., 2009). Younger healthcare workers reported being happier (Prizmic et al., 2009).

Determinants of healthcare professionals happiness in the intervention studies. The four intervention studies examined in this review included $\sim 11.9 \%(n=418 / 3501)$ of the healthcare professionals in this review. The healthcare professionals represented included medical surgical nurses (Appel et al., 2013), oncologists (Clemons et al., 2019), and healthcare workers'/professionals in general (Sexton and Adair, 2019; Coo and Salanova, 2018). Like the cross-sectional studies, the intervention studies all used self-report measures to assess happiness, namely, the Happiness Measure Questionnaire, the OHQ, the PHI and the SHS. A summary of the intervention studies on healthcare professionals happiness in this systematic review is presented in Table 2.

In North America (specifically in the USA and Canada), three studies assessed happiness following different interventions. In a study among medical surgical nurses in the USA $(n=91)$, no statistically significant differences in general happiness scores were found between the control group $(n=43)$ and group that completed an intervention involved the impact of journaling on perceived happiness $(n=48)$ (Appel et al., 2013). However, the variance in the quality of care provided by the nurses was explained by baseline happiness scores both pre- and postintervention (Appel et al., 2013). Additionally, in the USA, another study among healthcare workers $(n=280)$ who completed the Three Good Things intervention revealed increases in subjective happiness over time (Sexton and Adair, 2019). In a Canadian study among oncologists $(n=13)$, an intervention involving a 13week self-improvement virtuous program led to no statistically significant improvements in oncologists' happiness (Clemons et al., 2019) (see Table 2).

In Spain, a trial involving a mindfulness-based intervention (MBI) with three 150-min sessions among healthcare workers $(n=34)$ (intervention group $n=19$ and control group $=15$ ) was carried out (Coo and Salanova, 2018). Both interaction and time effects of the intervention and control groups were reported (Coo and Salanova, 2018). Scholars reported that the MBI program of three (150-min) sessions led to an increase in happiness among healthcare workers (Coo and Salanova, 2018).

Determinants of healthcare professionals happiness in the qualitative studies. The three qualitative studies reviewed herein included $\sim 2 \% \quad(n=69 / 3501)$ of the healthcare professionals. Across the three qualitative studies, a total of 18 determinants of healthcare professionals happiness were reported (as shown in Table 3). Among the three studies were two ethnographic and one narrative inquiry.

An ethnographic study among pediatricians and nursing personnel $(n=40)$ in the NICU in Iceland identified approximately four considerable emerging determinants of happiness (Einarsdottir, 2012). The determinants of healthcare professionals happiness in the NICU reported include the opportunity to do good via the provision of professional healthcare to children, professional pride, love for the well-being of the children, and a belief that happiness supersedes ethical dilemmas.

In the USA, another ethnographic study among physiotherapists $(n=15)$ reported four substantial determinants of physiotherapist happiness (Gannotti et al., 2019). The determinants of the physiotherapists' happiness included feeling and believing that one's work is meaningful and purposeful, the chance to be altruistic by helping the patients learn new and healthy skills to increase their independence, having an opportunity for quality 


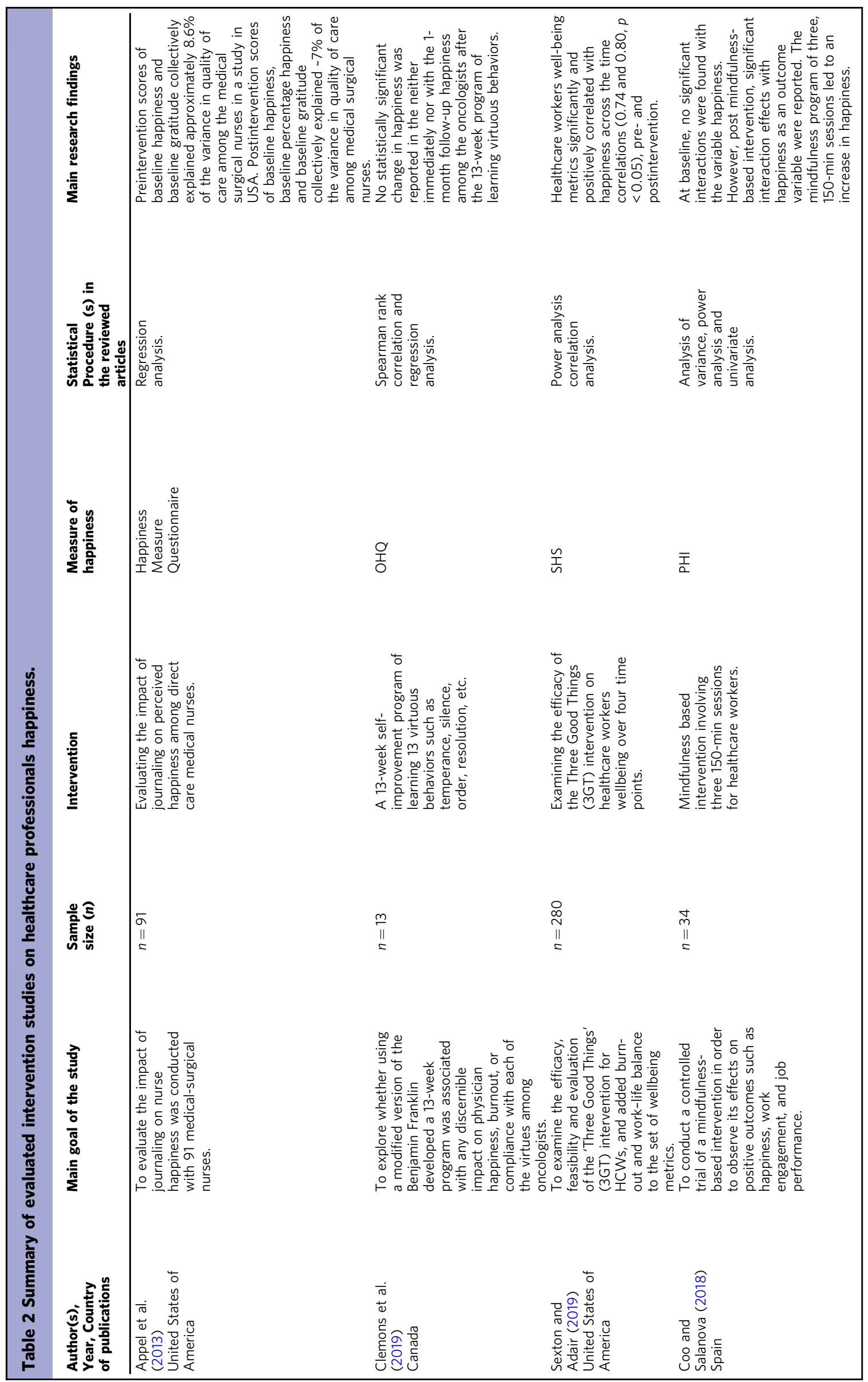




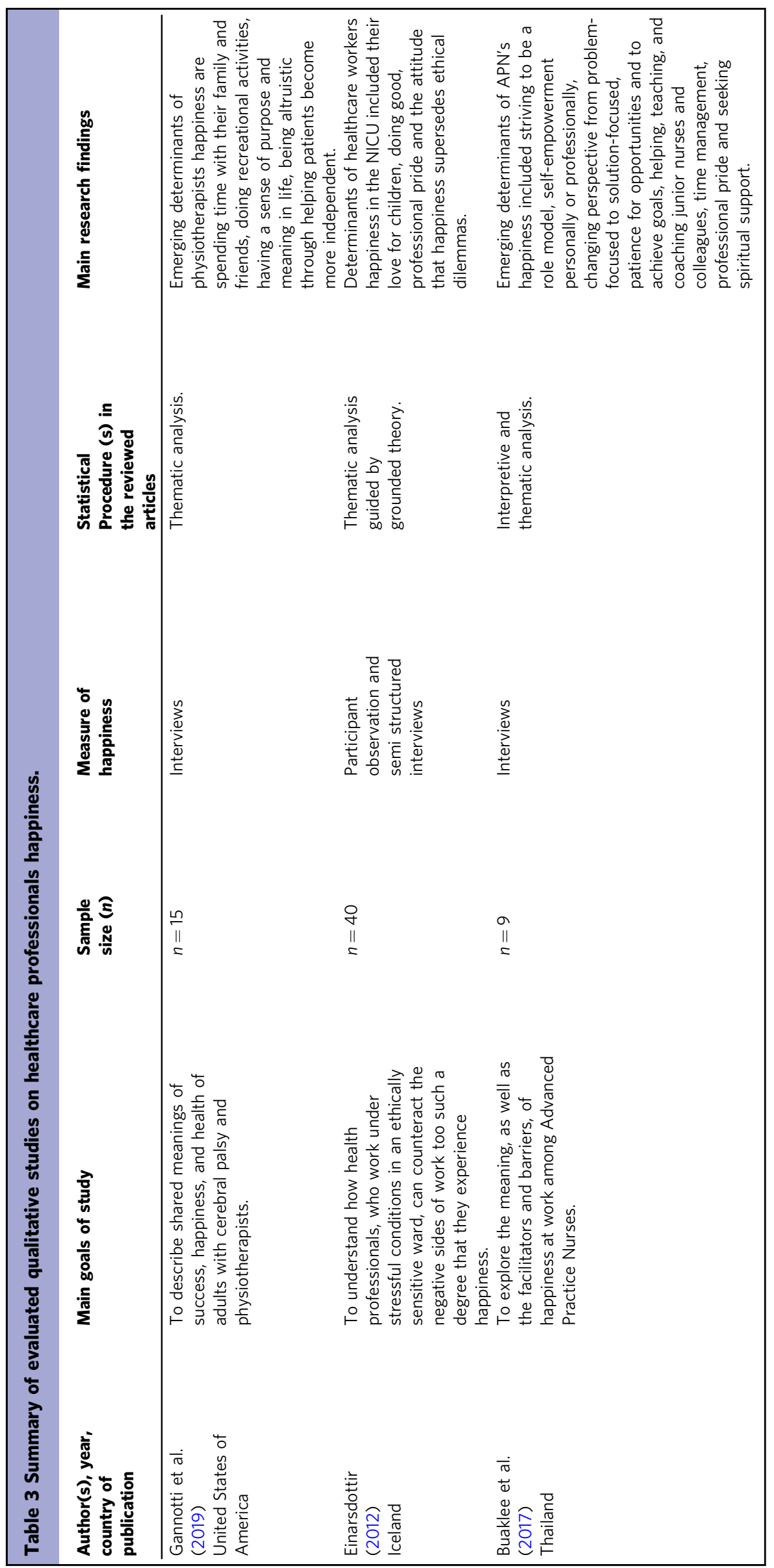


Table 4 Methodological quality of studies included.

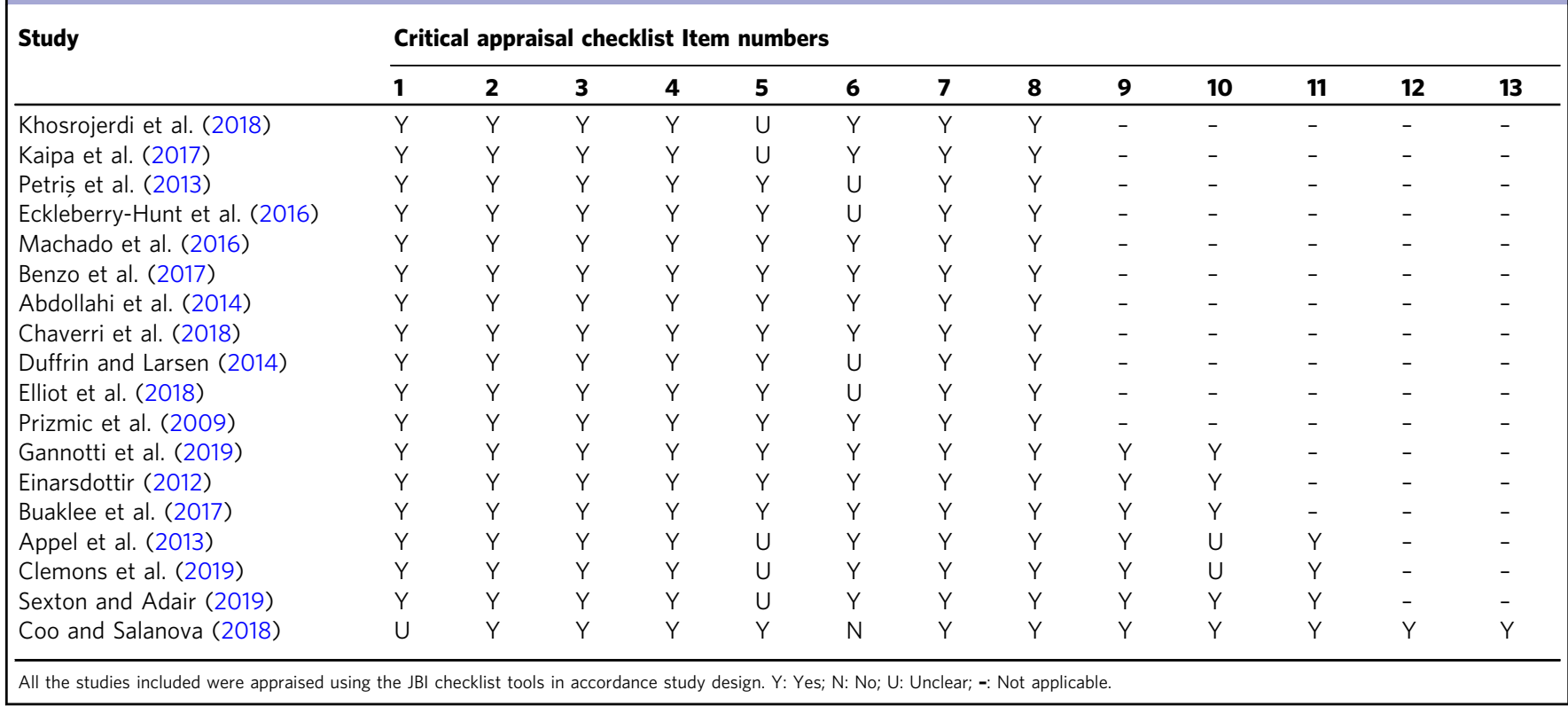

time with family and friends and leisure time to do recreational activities (Gannotti et al., 2019).

The third qualitative study performed in Thailand involved the qualitative research design known as narrative inquiry. From a total of nine advanced practice nurses, 10 substantial determinants of happiness were reported among APNs (Buaklee et al., 2017) (see Table 3). The determinants that led to increased happiness among APNs were being more optimistic by changing one's perspective from problem-focused to solution-focused, engaging in self-empowerment personally or professionally, striving to be a role model, time management, professional pride, exercising patience as one seeks opportunities to achieve their professional goals, helping, teaching, and coaching junior nurses and colleagues, and seeking spiritual support.

\section{Discussion}

In this systematic review, we aimed to examine the individualand organization-level determinants of healthcare professionals happiness globally between 2009 and 2019. Happiness is the degree to which an individual enjoys his or her present life (Veenhoven, 2017). The happier healthcare professionals are, the better the quality of health care and services they provide (Khosrojerdi et al., 2018; Kaipa et al., 2017). Since happiness is an attitudinal concept, we cannot measure it solely based on observing behavior; instead, assessments are a better way of comprehensively measuring the subjective delight of one's life (Veenhoven, 2017). Thus, the studies reviewed herein applied different forms of questioning, such as self-report measures and interviews. The determinants of happiness reported in this review indicate the roles of the individual and organizations in the happiness of healthcare professionals.

Individual determinants of healthcare professionals happiness. In this review, individual determinants are the inimitable factors that contribute to healthcare professionals happiness. From the research findings reported in this review, individual determinants refer to the role of attitudes, perceptions, knowledge and skills on healthcare professionals happiness.

Age is an individual determinant of happiness and was reported in four studies with differing outcomes (Khosrojerdi et al., 2018; Petriş et al., 2013; Kaipa et al., 2017; Prizmic et al., 2009). Among Romanian cardiologists, older cardiologists (specifically those above 65 years) were happier (Petriş et al., 2013), which was similar to the findings among dentists from India (Kaipa et al., 2017). However, in Croatia, the opposite was true; the younger healthcare workers were, the happier they were (Prizmic et al., 2009). These differences could be attributed to contextual factors. Although both countries are in Europe, they are different countries, and cultural differences cannot entirely be dismissed. In addition, the period during which the studies were performed could influence the results. For example, the two studies reported findings in two years, i.e., 2009 in Croatia (Prizmic et al., 2009) and 2013 in Romania (Petriş et al., 2013), thus partially explaining the varied findings on the phenomenon of age and happiness.

Gender is a social construct that defines and describes human behavior in terms of perceptions of masculinity and femininity (Muthuri, 2016). Gender differences among healthcare professionals happiness were reported in two cross-sectional studies. In the USA, for instance, female fellowship graduate physicians were happier than their male counterparts (Duffrin and Larsen, 2014). In India, among dentists, males are happier than their female counterparts (Kaipa et al., 2017). This could be attributed to cultural perceptions of masculinity and femininity in the different countries. According to Hofstede, masculinity and femininity are based on societal perceived values assigned between males and females, which vary across cultures and contexts (Hofstede, 2011). To create and effectively implement factual and relevant happiness policies, collaboration between healthcare professionals, researchers and policy makers is paramount. The collaboration in the research capturing, the perceptions of gender and how it impacts healthcare professionals happiness could be done across different contexts, either regional, national or rural versus urban.

The results of the present review reveal the role of attitude on healthcare professionals happiness. Attitude is both cognitive and affective in nature and has been found to influence health behavioral change (Lawton et al., 2009). A positive attitude is a signature strength that contributes to authentic happiness (Seligman, 2004). In this review, adopting positive attitudes resulted in increased happiness among healthcare professionals. 
A positive attitude involving self-empowering attitudes, adopting the attitude that happiness supersedes ethical dilemmas, allowed healthcare professionals to view challenges from a problem to a solution-focused point (Buaklee et al., 2017; Einarsdottir, 2012). A positive attitude among healthcare professionals allowed them to achieve a sense of purpose and personal accomplishment (Elliot et al., 2018; Eckleberry-Hunt et al., 2016; Seligman, 2004; Peterson et al., 2005).

Acquiring a sense of meaning and purpose in one's life has been reported to result in the long-term experience of happiness (Peterson et al., 2005). As a result, healthcare professionals professional pride allows them to strive to be role models for their colleagues (Einarsdottir, 2012; Buaklee et al., 2017). The more compassionate healthcare professionals were to themselves, the happier they were (Benzo et al., 2017). Thus, policy makers should not only emphasize compassion towards others but also self-compassion, thus enabling individuals to provide compassion to others. Generosity is another signature strength (Seligman, 2004), referred to as altruism in this review; this trait contributed to healthcare professionals happiness.

Altruism was a universal emerging theme across the qualitative studies in this systematic review. Altruistic behavior involves helping another individual enhance their welfare (Muthuri, 2018). In the WHR 2012, altruism was one of the causes of increased happiness, implying that the more altruistic an individual is, the happier they are (Richard Layard and Claudia, 2012). The healthcare professionals reported finding happiness by helping patients learn skills to enhance their independence (Gannotti et al., 2019), doing good by helping preterm infants and their parents during the challenging times (Einarsdottir, 2012), and helping colleagues by coaching and teaching new skills and techniques to enhance their performance (Buaklee et al., 2017). The nature of healthcare professions involves having a desire to help others effectively perform and provide quality healthcare. Health authorities could provide opportunities for healthcare professionals to express their generosity or altruism, which has been found to increase happiness. For example, creating mentorship programs recognizable within health facilities and the health system to encourage altruistic behavior could result in increased happiness among healthcare professionals.

Researchers have suggested future research and policy to shift focus from mental illness to mental health (Seligman and Csikszentmihalyi, 2000). In this review, satisfaction with mental health was a determinant of happiness to increase happiness healthcare professionals cope in various ways, such as by perceiving stress as minimal and using defense mechanisms such as optimistic anticipation and humor (Abdollahi et al., 2014; Eckleberry-Hunt et al., 2016; Khosrojerdi et al., 2018; Machado et al., 2016). These findings highlight the importance of implementing programs promoting mental health strategies. Strategies related to generating healthy coping mechanisms and increasing happiness among healthcare professionals to optimize their productivity at an individual and organizational level.

Quality of life is a cognitive concept that is used as a basis for lifestyle decision making based on an individual's perception of consequences (Susniene and Jurkauskas, 2009). As constructs, happiness and quality of life are closely associated but are not identical (Susniene and Jurkauskas, 2009). In this review, quality of life was a determining factor of healthcare professionals happiness. Some decisions, such as participating in recreational activities, taking vacations and breaks, spending quality time with their friends and family, and good quality sleep, are related to quality of life among healthcare professionals, thus contributing to their happiness (Khosrojerdi et al., 2018; Petriş et al., 2013; Machado et al., 2016; Gannotti et al., 2019). Policies and programs allowing for enhanced quality of life can enable healthcare professionals to revitalize and reenergize themselves through some of the ways reported in this systematic review. The examination of the individual determinants of healthcare professionals happiness illustrates the role of individuals in their own happiness as well as the role of organizations they work for.

Organizational determinants of healthcare professionals happiness. Organizational determinants of healthcare professionals happiness are related to organization dynamics, structure and behavior within health facilities and/or health systems at large.

In 2008, researchers stated that not only can career success result in happiness but also happiness can result in career/ workplace success (Boehm and Lyubomirsky, 2008). Career development, specifically vocational identity achievement, has been associated with pursuit engagement and meaning as orientations of happiness (Hirschi, 2011). Career development is a progressive process involving the diversification of oneself through various actions to acquire better knowledge and skills. Findings from this review showed that healthcare professionals career development was a determinant of happiness; through increasing their work experience, acquiring higher qualifications, being both clinicians and academicians, were some of the career development and success strategies that contributed to the healthcare professionals happiness (Kaipa et al., 2017; Petriş et al., 2013). This implies that opportunities for career development within health organizations and health systems need to be considered when developing happiness policies and strategies.

Among healthcare professionals in this review, time management, workload manageability, satisfaction with income, satisfaction with colleagues conduct and performance, having more sessions with clients, job rotation in the hospital wards and shifts were among the organizational dynamics that contributed to their happiness (Buaklee et al., 2017; Eckleberry-Hunt et al., 2016; Khosrojerdi et al., 2018). The more satisfaction one experiences both at work and in life, the happier they are likely to be and the better they are likely to perform (Argan et al., 2018; Streimikiene and Grundey, 2009). Therefore, policy makers should not only consider financial satisfaction but also enhance job satisfaction by improving organizational culture and dynamics.

Intervention studies such as other types of studies provide insight into promising strategies through developing researchbased capacity-building interventions in policy settings (Haynes et al., 2018). In this review, two other studies reported a significant improvement in healthcare professionals happiness using two interventions, namely, the Three Good Things intervention (3GT) (Sexton and Adair, 2019) and the Mindfulness Based Intervention (MBI) (Coo and Salanova, 2018). However, another two intervention studies showed no significant improvement in healthcare professionals happiness when using journaling as an intervention (Appel et al., 2013) or the 13virtuous self-improvement program (Clemons et al., 2019). The findings from this review suggest that some interventions could work, and others may not. The success or failure of an intervention is based on its interaction with the context (Haynes et al., 2018). An ideal intervention involves components such as contextually based interventions, goals and genuine collaboration of persons and partners (Haynes et al., 2018). Therefore, health policy makers and researchers could consider adopting an open mindset when exploring the effectiveness of happiness interventions among populations such as healthcare professionals in varied organizational contexts.

Work-life balance involves taking actions towards effectively managing the demands and responsibilities of both work and life (such as the self, family and friends) (Otken and Erben, 2013). 
The opposite of work-life balance is work-life conflict. As a result of work-life conflict, some organizations chose to develop policies and strategies geared towards empowering their employees to achieve work-life balance (Otken and Erben, 2013). In this review, achieving work-life balance was a determinant of happiness among healthcare professionals balance (Petriş et al., 2013; Machado et al., 2016; Elliot et al., 2018). In Turkey, research has reported that the happiness of employees increases when they are driven both in their work and life domains (Otken and Erben, 2013). Therefore, evidence-based policies and strategies geared towards energizing and empowering healthcare professionals to feel better at home and work are significant in increasing their happiness.

Physical health is paramount, but people also strive for happiness, sense of meaning and purpose and being a good person with healthy relationships (VanderWeele et al., 2020). All these factors contribute to a healthy and full life. A study based on global data on happiness found that the greater happiness, the better health and longevity the population is likely to experience, thus reflecting human flourishing (Veenhoven, 2017). In this review, exercising and having leisure time to perform recreational activities were determinants of healthcare professionals happiness (Gannotti et al., 2019; Benzo et al., 2017). In this review, it is evident that healthcare professionals are human beings who strive to be healthy and need physical, psychological, and social support to be healthier and happier.

Policies and implementation of strategies geared towards training healthcare professionals holistic development are essential. Interventions empowering healthcare professionals to enhance the synergy between their intrapersonal and interpersonal development are paramount for their happiness. From an individual standpoint, happiness is a result of being in harmony with one's life (Streimikiene and Grundey, 2009). Based on evidence in this review, individual determinants of healthcare professionals happiness do not operate mutually exclusively from the organizational determinants. Healthcare professionals happiness is a result of an interplay between the healthcare professionals (as individuals) and the organization (health facilities and health system). In the research area of healthcare professionals happiness, there are knowledge gaps that still exist. In the following section, we identify limitations and research gaps in the current literature to inform future research on healthcare professionals happiness.

Limitations and recommendations for future studies. Various limitations were noted in this review, which are possible avenues for further research. The most cited limitation was a small sample size across both quantitative and qualitative studies (Buaklee et al., 2017; Gannotti et al., 2019; Coo and Salanova, 2018; Clemons et al., 2019; Duffrin and Larsen, 2014; Chaverri et al., 2018; Machado et al., 2016; Petriş et al., 2013). According to researchers, a small sample size results in lower power for statistical comparison (Gannotti et al., 2019), an inability to generalize results (Abdollahi et al., 2014; Coo and Salanova, 2018; Machado et al., 2016), seemingly erroneous data (Clemons et al., 2019), sample bias (Elliot et al., 2018; Abdollahi et al., 2014), selection bias (Chaverri et al., 2018; Eckleberry-Hunt et al., 2016; Kaipa et al., 2017), response bias (Abdollahi et al., 2014; Benzo et al., 2017), and low response rates (Duffrin and Larsen, 2014).

While larger samples were highly recommended, the same is true for heterogeneous samples; future studies should include various groups of healthcare professionals (Coo and Salanova, 2018; Clemons et al., 2019; Prizmic et al., 2009; Elliot et al., 2018; Duffrin and Larsen, 2014; Benzo et al., 2017; Machado et al., 2016; Eckleberry-Hunt et al., 2016). Some researchers pointed out that heterogeneous sampling is essential in cases where there is no gender balance or when there is an overrepresentation of a healthcare profession (Sexton and Adair, 2019). Researchers recommend that future studies consider using complementary approaches or multidimensional approaches when assessing happiness both methodologically and theoretically to provide a comprehensive understanding of the concept of happiness among healthcare professionals (Einarsdottir, 2012; Coo and Salanova, 2018; Buaklee et al., 2017).

Additional limitations included high attrition rates in intervention studies (Coo and Salanova, 2018); inaccurately completed questionnaires or answers, some of which were attributed to limited understanding of a concept (Abdollahi et al., 2014; Buaklee et al., 2017); measurement error due to the use of only self-report assessments that could result in positive or negative tendencies (Kaipa et al., 2017; Coo and Salanova, 2018); and the timing of the studies, as receiving negative news on disasters could affect individuals' happiness (Appel et al., 2013).

In addition, more comparative studies between healthcare professionals happiness and other psychological factors are needed (Abdollahi et al., 2014), as well as studies on the role of organizational culture on the quality of services provided by healthcare professionals (Coo and Salanova, 2018). Other behavioral indicators should also be used, such as ratings from a second person and not solely focusing on self-report measures (Coo and Salanova, 2018). Similar studies should be done in various settings (Buaklee et al., 2017; Duffrin and Larsen, 2014) to discover the impact of happiness on health systems in different countries across the seven continents. Last, longitudinal studies were recommended by various researchers to assess healthcare professionals happiness over time (Elliot et al., 2018).

\section{Conclusions}

In this review, the authors examined the role of healthcare professionals (as individuals) and organizations (health facilities and health systems) in determining healthcare professionals happiness. Happiness is a significant component of healthcare professionals optimal functioning. Based on an examination of recent literature, it is evident that the individual and organizational determinants are not mutually exclusive but interdependently function to achieve healthcare professionals happiness. Thus, ideal and effective happiness policies and implementation strategies need to apply a collaborative approach between healthcare professionals and health authorities at the organization and health system levels. Health policy makers should consider continuously increasing the happiness of healthcare professionals, as it has a significant role in improving the quality of healthcare delivery and health outcomes within health systems. By promoting evidence-based contextually relevant healthcare professionals happiness policies, programs and strategies, health workforce strengthening, and health system strengthening are likely to occur. This review sets the stage for subsequent research contributions aimed at promoting healthcare professionals happiness. Based on the research gaps highlighted in this study, more research on healthcare professionals happiness is needed across all continents. Thus, researchers should explore and contribute to bridging the knowledge gaps regarding healthcare professionals happiness in various contexts.

\section{Data availability}

All the data (reviewed articles) used, were from already published empirical articles, retrieved from databases. All data generated or analyzed during this study are included in this published article. 
Received: 25 April 2020; Accepted: 3 September 2020;

Published online: 16 September 2020

\section{Note}

1 https://www.crd.york.ac.uk/prospero/display_record.php?ID=CRD42020145855

\section{References}

Abdollahi A, Abu Talib M, Yaacob SN, Ismail Z (2014) Hardiness as a mediator between perceived stress and happiness in nurses. J Psychiatr Ment Health Nurs 21:789-796

Anand S, Bärnighausen T (2012) Health workers at the core of the health system: framework and research issues. Health Policy 105:185-191

Appel L, Labhart L, Balczo P, Mccieary N, Raley M, Winsett RP (2013) A comparative study of a happiness intervention in medical-surgical nurses. MEDSURG Nurs 22:319-324

Argan M, Argan MT, Dursun MT (2018) Examining relationships among wellbeing, leisure satisfaction, life satisfaction, and happiness. Int J Med Res Health Sci 7:49-59

Argyle M, Martin M, Crossland J (1989) Happiness as a function of personality and social encounters. In Forgas JP, Innes JM (Eds.), Recent advances in social psychology: An international perspective, 189-203. North-Holland: Elsevier Science Publishers B.V.

Atkins D, Fink K, Slutsky J, Agency for Healthcare R, Quality \& North American Evidence-based Practice C (2005) Better information for better health care: the Evidence-based Practice Center program and the Agency for Healthcare Research and Quality Annals of internal medicine 142:1035-1041

Benzo RP, Kirsch JL, Nelson C (2017) Compassion, mindfulness, and the happiness of healthcare workers. Explore (New York, NY) 13:201-206

Berkland BEMA, Werneburg BLBA, Jenkins SMMS, Friend JLBS, Clark MMP, Rosedahl JKBS, Limburg PJMD, Riley BAMBA, Lecy DRBS, Sood AMD (2017) A worksite wellness intervention: improving happiness. Life satisfaction, and gratitude in health care workers Mayo Clin Proc Innov Qual Outcomes 1(3):203-210

Boehm JK, Lyubomirsky S (2008) Does happiness promote career success? J Career Assess 16:101-116

Brand SL, Thompson Coon J, Fleming LE, Carroll L, Bethel A, Wyatt K, Van Wouwe JP (2017) Whole-system approaches to improving the health and wellbeing of healthcare workers: a systematic review. PLoS ONE 12:e0188418

Buaklee P, Fongkaew W, Turale S, Akkadechanunt T, Sansiriphun N (2017) Unveiling the experiences of happiness at work through narrative inquiry: Advanced Practice Nurses' Perspectives. Pac Rim Int J Nurs Res 21:206-219

Chaverri J, Praetorius RT, Ruiz E (2018) Counselor happiness: effects of therapy work with similar trauma. Social Work in Mental Health 16:419

Clemons M, Mazzarello S, Pond G, Amir E, Asmis T, Berry S, Brackstone M, Brule S, Goodwin R, Hilton JF, Juliao M, Nicholas G, Stewart DJ, Wheatley-Price P, Cholmsky L, Krentel A, Hutton B, Joy AA (2019) A prospective intervention to improve happiness and reduce burnout in oncologists. Support Care Cancer 27:1563-1572

Coo C, Salanova M (2018) Mindfulness can make you happy-and-productive: a mindfulness controlled trial and its effects on happiness, work engagement and performance. J Happiness Stud 19:1691-1711

Duffrin C, Larsen L (2014) The effect of primary care fellowship training on career satisfaction, happiness and perceived stress. Postgrad Med J 90:377-82

Dyrbye LN, Shanafelt TD, Sinsky CA, Cipriano PF, Bhatt J, Ommaya A, West CP, Meyers D (2017) Burnout among health care professionals: a call to explore and address this underrecognized threat to safe, high-quality care. NAM (Natl Acad Med) Perspect. Discussion Paper 1-11. [online]. https://nam.edu/ burnout-among-health-care-professionals-a-call-to-explore-and-addressthis-un-derrecognized-threat-to-safe-high-quality-care

Eckleberry-Hunt J, Kirkpatrick H, Taku K, Hunt R, Vasappa R (2016) Relation between physicians' work lives and happiness. South Med J 109:207-212

Einarsdottir J (2012) Happiness in the neonatal intensive care unit: merits of ethnographic fieldwork. Int J Qual Stud Health Well-being 7:1-9

Elliot M, Cullen M, Calitz AP (2018) Happiness among South African private sector physiotherapists. South Afr J Physiother 74:1-10

Gannotti ME, Blanchard Y, Blumberg L, Larocco D (2019) Shared meanings of success, happiness, and health among adults with cerebral palsy and physiotherapists: implications for practice and research. Disabil Rehabil 41:1321-1330

Haynes A, Rowbotham SJ, Redman S, Brennan S, Williamson A, Moore G (2018) What can we learn from interventions that aim to increase policy-makers' capacity to use research? A realist scoping review. Health Res Policy Syst 16:31

Hervás G, Vázquez C (2013) Construction and validation of a measure of integrative well-being in seven languages: the Pemberton Happiness Index. Health Qual Life Outcomes 11:66
Hills P, Argyle M (2002) The Oxford Happiness Questionnaire: a compact scale for the measurement of psychological well-being. Pers Individ Differ 33:1073-1082

Hirschi A (2011) Effects of orientations to happiness on vocational identity achievement. Career Dev Q 59:367-378

Hofstede G (2011) Dimensionalizing cultures: the Hofstede model in context. Online Read Psychol Cult 2:2307-0919.1014

Huang PH (2010) Happiness studies and legal policy. Annu Rev Law Soc Sci 6:405-432

Jan-Emmanuel De Neve GW (2017). Chapter 6: Happiness at work. World Happiness Report 2017, p. 34

Joanna Briggs Institute. Critical appraisal tools [online]. https://joannabriggs.org/ critical_appraisal_tools. Accessed 4 Aug 2019

Joshanloo M, Park YO, Park SH (2017) Optimism as the moderator of the relationship between fragility of happiness beliefs and experienced happiness. Personal Individ Differ 106:61-63

Kaipa S, Paul KK, Satpathy A, Epari V (2017) Are dentists happy? A study among dental practitioners in coastal Andhra Pradesh using subjective happiness scale. Indian J Dent Res 28:604-608

Khosrojerdi Z, Tagharrobi Z, Sooki Z, Sharifi K (2018) Predictors of happiness among Iranian nurses. Int J Nurs Sci 5:281-286

Kokonya DA, Mburu JM, Kathuku DM, Adam AH, Nshimirimana DA, Biraboneye PS, Kpoto LM (2014) Burnout syndrome among medical workers at Kenyatta National Hospital (KNH), Nairobi, Kenya. J Psychiatry 17:14-32

Lafreniere JP, Rios R, Packer H, Ghazarian S, Wright SM, Levine RB (2016) Burned out at the bedside: patient perceptions of physician burnout in an internal medicine resident continuity clinic. J Gen Intern Med 31:203-208

Lawton R, Conner M, Mceachan R (2009) Desire or reason: predicting health behaviors from affective and cognitive attitudes. Health Psychol 28:56-65

Liberati A, Altman DG, Tetzlaff J, Mulrow C, Gøtzsche PC, Ioannidis JPA, Clarke M, Devereaux PJ, Kleijnen J, Moher D (2009) The PRISMA statement for reporting systematic reviews and meta-analyses of studies that evaluate healthcare interventions: explanation and elaboration. BMJ 339: b2700

Lyubomirsky S, Lepper HS (1999) A measure of subjective happiness: preliminary reliability and construct validation. Soc Indic Res 46:137-155

Lyubomirsky S, Sheldon KM, Schkade D (2005) Pursuing happiness: the architecture of sustainable change. Rev Gen Psychol 9:111-131

Machado L, Tavares H, Petribu K, Pinto T, Cantilino A (2016) Happiness and defense styles in psychiatrists. J Nerv Ment Dis 204:181-187

Moher D, Shamseer L, Clarke M, Ghersi D, Liberati A, Petticrew M, Shekelle P, Stewart LA (2015) Preferred Reporting Items for Systematic Review and Meta-Analysis Protocols (PRISMA-P) 2015 statement. Syst Rev 4:1

Muthuri RNDK (2016) Self-concept among university students: a case study of the United States International University in Africa. Lambert Academic Publishing, Saarbrucken

Muthuri RNDK (2018) Predictors of altruistic behaviour among a sample of emerging adults at the United States International University in Africa. Ann Behav Sci 4:2. https://doi.org/10.21767/2471-7975.100035

Otken A, Erben GS (2013) The relationship between work-life balance and happiness from the perspectives of generation X and Y. Humanit Soc Sci Rev $2: 45-53$

Paiva CE, Paiva BSR, De Camargos MG, Demarzo MMP, Hervas G, Vázquez C (2016) The pemberton happiness index: validation of the Universal Portuguese version in a large Brazilian sample. Medicine 95:e4915

Peterson C, Park N, Seligman ME (2005) Orientations to happiness and life satisfaction: the full life versus the empty life. J Happiness Stud 6:25-41

Petriș A, Tatu-Chițoiu G, Pop C (2013) Highlights on Romanian cardiologist's happiness: results of fellow cardiologists' self-rating. Romanian J Cardiol 23:3-11

Pollock NC, Noser AE, Holden CJ, Zeigler-Hill V (2016) Do orientations to happiness mediate the associations between personality traits and subjective well-being? J Happiness Stud 17:713-729

Prizmic Z, Lipovcan LK, Burusic J (2009) Off-the-job activities and well-being in healthcare professionals. Rev Za Soc Politiku 16:271-280

Richard Layard AC, Claudia S (2012) World Happiness Report 2012: Chapter 3. The causes of happiness and misery

See KF, Yen SH (2018) Does happiness matter to health system efficiency? A performance analysis. Health Econ Rev 8:33

Seligman ME (2004) Authentic happiness: using the new positive psychology to realize your potential for lasting fulfillment. Simon and Schuster

Seligman ME, Csikszentmihalyi M (2000) Positive psychology: an introduction. Am Psychol Assoc 55:5-14

Sexton JB, Adair KC (2019) Forty-five good things: a prospective pilot study of the Three Good Things well-being intervention in the USA for healthcare worker emotional exhaustion, depression, work-life balance and happiness. BMJ Open 9:e022695 
Shojania KG, Sampson M, Ansari MT, Ji J, Doucette S, Moher D (2007) How quickly do systematic reviews go out of date? A survival analysis. Ann Intern Med 147:224-233

Streimikiene D, Grundey D (2009) Life satisfaction and happiness-the factors in work performance. Econ Sociol 2:9

Susniene D, Jurkauskas A (2009) The concepts of quality of life and happinesscorrelation and differences. Eng Econ 63:58-66

United Nations (2012) Resolution adopted by General Assembly on 28 June 2012 $66 / 281$. International Day of Happiness, A/RES/66/281. United Nations, Geneva

Vanderweele TJ, Chen Y, Long K, Kim ES, Trudel-Fitzgerald C, Kubzansky LD (2020) Positive Epidemiology? Epidemiology 31:189-193

Veenhoven R (2017) Greater happiness for a greater number: did the promise of enlightenment come true? Soc Indic Res 130:9-25

Walsh LC, Boehm JK, Lyubomirsky S (2018) Does happiness promote career success? Revisiting the evidence. J Career Assess 26:199-219

Whiting P, Savović J, Higgins JP, Caldwell DM, Reeves BC, Shea B, Davies P, Kleijnen J, Churchill R (2016) ROBIS: a new tool to assess risk of bias in systematic reviews was developed. J Clin Epidemiol 69:225-234

World Health Organization (2007) Everybody's business: strengthening health systems to improve health outcomes. WHO'S framework for action [Online]. https://www.who.int/healthsystems/strategy/everybodys_business.pdf

World Health Organization (2013) Transforming and scaling up health professionals education and training: World Health Organization Guidelines 2013 [Online]. World Health Organization, Geneva. https://www.ncbi.nlm.nih. gov/books/NBK298950/

\section{Acknowledgements}

The authors thank Estelle Grobler of the Medical School Library at the University of Pretoria, for her support as an information specialist during our comprehensive systematic search across the databases. We are deeply grateful to God for meeting all our needs during the entire process of the study reported in this paper.

\section{Author contributions}

All authors contributed substantially to this paper. All authors read and approved the final version of this manuscript for publication.

\section{Competing interests}

The authors declare no competing interests.

\section{Additional information}

Correspondence and requests for materials should be addressed to R.N.D.K.M.

Reprints and permission information is available at http://www.nature.com/reprints

Publisher's note Springer Nature remains neutral with regard to jurisdictional claims in published maps and institutional affiliations.

\begin{abstract}
(c) (i) Open Access This article is licensed under a Creative Commons Attribution 4.0 International License, which permits use, sharing, adaptation, distribution and reproduction in any medium or format, as long as you give appropriate credit to the original author(s) and the source, provide a link to the Creative Commons license, and indicate if changes were made. The images or other third party material in this article are included in the article's Creative Commons license, unless indicated otherwise in a credit line to the material. If material is not included in the article's Creative Commons license and your intended use is not permitted by statutory regulation or exceeds the permitted use, you will need to obtain permission directly from the copyright holder. To view a copy of this license, visit http://creativecommons.org/ licenses/by/4.0/.
\end{abstract}

(C) The Author(s) 2020 